\title{
Uma Proposta para o Desenvolvimento do Pensamento Computacional Integrado ao Ensino de Matemática*
}

\author{
Mônica Marques ${ }^{1}$, Simone Cavalheiro ${ }^{1}$, Luciana Foss ${ }^{1}$, \\ Adriana Bordini ${ }^{1}$, Christiano Ávila ${ }^{1}$ \\ ${ }^{1}$ Programa de Pós-Graduação em Computação - Universidade Federal de Pelotas \\ CEP 96.010-610 - Pelotas- RS - Brazil \\ \{mmd. cunha, simone. costa, lfoss\}@inf.ufpel.edu.br
}

\begin{abstract}
The introduction of the fundamentals of Computing in Basic Education has been focus of attention and discussion in recent years. In particular, Computational Thinking (CT) has become popular in the last decade as a methodology capable of developing many of the skills needed to solve problems. Concurrently, the relationship of bidirectional support between PC and Mathematics has been already discussed, in the sense that several of the skills to be developed in the different areas intersect or even complement each other. This work establishes a correlation between the areas and proposes a conceptual model for the proposition of mathematical activities that jointly promote the development of PC skills, focusing on the initial series of Primary Education.
\end{abstract}

Resumo. A introdução dos fundamentos da Computação na Educação Básica tem sido foco de atenção e discussão nos últimos anos. Em particular, o Pensamento Computacional (PC) se popularizou como uma metodologia capaz de desenvolver muitas das habilidades necessárias à resolução de problemas. Também, já se discute a relação de apoio bidirecional entre o PC e a Matemática, no sentido que diversas das habilidades a serem desenvolvidas nas diferentes áreas se interseccionam ou mesmo se complementam. Este trabalho estabelece uma correlação entre as áreas e propõe um modelo conceitual para a proposição de atividades matemáticas que impulsiona o desenvolvimento de habilidades do PC, com foco nas séries iniciais da Educação Fundamental.

\section{Introdução}

A introdução dos fundamentos da Computação na Educação Básica tem sido foco de atenção e discussão nos últimos anos [da Silva Rodrigues et al. 2015, Bordini et al. 2016], como consequência natural da globalização e dos avanços tecnológicos. Particularmente, o Pensamento Computacional (PC) [Wing 2006] se popularizou na última década como uma metodologia que "envolve a resolução de problemas, o projeto de sistemas e a compreensão do comportamento humano, recorrendo aos conceitos fundamentais da Ciência da Computação".

Concomitantemente, já se discute a relação de apoio bidirecional entre o PC e a Matemática [Lewis and Shah 2012, Barcelos and Silveira 2012], no sentido que diversas das habilidades a serem desenvolvidas nas diferentes áreas

\footnotetext{
* Projeto com chancela da SBC e realizado com o apoio da PREC e PRPPG / UFPel.
} 
VI Congresso Brasileiro de Informática na Educação (CBIE 2017)

Anais do XXVIII Simpósio Brasileiro de Informática na Educação (SBIE 2017)

se interseccionam ou mesmo se complementam. Nos últimos anos, diversos trabalhos [Lu and Fletcher 2009, Freudenthal et al. 2010, da Costa Barbosa et al. 2015, Love et al. 2016, Lopes et al. 2016] propuseram a aproximação entre estas áreas, predominantemente, por meio da introdução de conceitos da Computação na disciplina de Matemática. Muitos destes estudos integram a programação ao ensino de Matemática (vários destes adotando a ferramenta Scratch [Prater and Mazur 2014, Bean et al. 2015]). Em especial, observa-se diversas tentativas (isoladas) de propostas de atividades de ensino que visam desenvolver habilidades do PC, apresentando conceitos ou utilizando ferramentas computacionais para a resolução de problemas matemáticos.

A integração entre diferentes áreas do conhecimento é um seguimento natural a concepção dos estudantes sobre a sua visão de realidade. Um dos principais motivos é porque as crianças não concebem o mundo como se fosse dividido em unidades separadas. Neste sentido, o conhecimento dever ser construído através da articulação de diferentes áreas curriculares, interpretando-as de forma globalizada e integrada e promovendo conexões adequadas e significativas [Barbosa and Vale 2014].

Este trabalho estabelece uma correlação entre a Computação e a Matemática e propõe um modelo conceitual para a proposição de atividades matemáticas que impulsiona, conjuntamente, o desenvolvimento de habilidades do PC, com foco nas séries iniciais da Educação Fundamental. Inicialmente, na Seção 2, é descrita uma inter-relação entre as competências da área de Matemática das séries iniciais com as competências do PC e então, na Seção 3, são definidos os principais fundamentos a serem considerados para a integração entre as áreas. $\mathrm{Na}$ Seção 4, um conjunto de orientações que guia a proposição de atividades de ensino de Matemática e estimula simultaneamente o desenvolvimento de habilidades do PC é delineado. As considerações finais estão na Seção 5.

\section{O Pensamento Computacional e o Currículo da Matemática para as Séries Iniciais no Brasil}

Esta seção discute a confluência entre a Matemática e o PC nas séries iniciais da Educação Básica, delineando uma inter-relação entre as competências a serem desenvolvidas nos estudantes nas respectivas áreas. O detalhamento leva em consideração os padrões da Ciência da Computação para o K-12 (referenciando-se educação primária e secundária dos EUA) estabelecidos pela CSTA (Computer Science Teachers Association) e ACM (Association for Computing Machinery) [CSTA and ACM 2016], bem como as competências e habilidades da disciplina de Matemática para as séries iniciais, detalhadas na Base Nacional Comum Curricular [Ministério da Educação 2016].

Nesta análise, o PC é considerado como um elemento central na disciplina (mais ampla) Ciência da Computação. Barcelos e Silveira, em [Barcelos and Silveira 2012], descrevem uma análise da confluência entre a Matemática e a Ciência da Computação (CC) na Educação Elementar. No que segue, estas relações (referentes a regularidades de sequencias e resoluções de problemas) são complementadas e novas são estabelecidas. Conforme discutido a seguir, observa-se que muitas das habilidades da área da Matemática podem ser tratadas como casos particulares de habilidades do PC.

Regularidades de Sequências, Reconhecimento de Padrões e Padrões de Processos: a identificação de regularidades, observação de regras de formação, determinação de elementos ausentes e construção de sequências repetitivas de números, objetos 
e figuras são habilidades a serem desenvolvidas na Matemática nas séries iniciais [Ministério da Educação 2016]. De forma mais ampla, o reconhecimento de padrões e elementos essenciais em modelos de objetos e processos de diferentes naturezas é foco do PC para o mesmo período [CSTA and ACM 2016]. A identificação de regularidades em processos de diversas áreas (como por exemplo, no ciclo da água, no ciclo de vida da borboleta, nos padrões climáticos sazonais) e a subsequente criação de modelos demanda estruturas e processos cognitivos que devem ser desenvolvidos. Quando objetos são números, as regras de regularidade são definidas por operações algébricas, tornando-se um caso particular do estudo de padrões de processos genéricos. Se a natureza do processo for diferente, a abstração dos objetos envolvidos não representam apenas quantidades ou figuras e precisam ser representados por estruturas adequadas que incluam as informações relevantes. A identificação e representação destas abstrações para diferentes tipos de processos são o foco do PC, que pode integrar o estudo das regularidades em sequências numéricas ou geométricas, como um caso particular. Habilidades de pensamento simbólico são necessárias para relacionar um modelo de um objeto com o artefato real (por exemplo, modelo de um carro a um carro real). A extração dos fatores essenciais de objetos (por exemplo, tamanho, forma, textura e função) são necessários para reconhecimento, discriminação, classificação e abstração. A criação de modelos de objetos com a finalidade de encontrar padrões e elementos essenciais (padrão da $\mathrm{CC}$ ) abrange o reconhecimento de figuras geométricas espaciais e suas respectivas associações com artigos do mundo físico (objetos de conhecimento da Geometria).

Resolução de Problemas e Algoritmos: a competência de ler e interpretar símbolos, códigos e nomes inerentes a linguagem matemática se interliga com a representação abstrata necessária à área de algoritmos. Por sua vez, algoritmos são modelos dinâmicos que podem ser integrados como um passo intermediário entre uma narrativa verbal (imprecisa e ambígua) e uma representação algébrica (formal e estática) [Barcelos and Silveira 2012]. Por exemplo, a relação linear entre quantidades pode ser introduzida numa equação algébrica (de forma estática) ou na definição e simulação de um algoritmo (de forma dinâmica). Alguns trabalhos [Lopes et al. 2016, Lewis and Shah 2012] já concluíram que o processo de construção de algoritmos auxilia no desenvolvimento de habilidades matemáticas, especialmente daquelas relacionadas com resolução de problemas. Habilidades da Matemática para os anos iniciais incluem a resolução de problemas envolvendo as operações aritméticas básicas (adição, subtração, multiplicação divisão). Por sua vez, o processo de definir e executar algoritmos (conjuntos de instruções passo-a-passo) que incluam sequenciamento e laços simples para realizar uma tarefa é um dos objetivos do PC. A definição de algoritmos para a realização das operações aritméticas básicas inclui diversos passos e níveis de abstração que podem contribuir de forma significativa na compreensão e sistematização das operações algébricas, abrangendo: a identificação de quais são as informações necessárias para a resolução dos problemas (entradas e seus tipos), qual o tipo de resultado esperado e, principalmente, quais são as etapas necessárias para, a partir da entrada, obter-se o resultado esperado. O esboço de roteiros e plantas é outro objeto de conhecimento da Matemática que pode ser 
enriquecido com a definição, simulação e análise de algoritmos.

Composição e Decomposição de Números, Problemas e Processos: a decomposição é um dos elementos centrais do PC. Ela é considerada como uma maneira de pensar sobre objetos ou processos em termos de suas partes componentes. As partes podem então ser compreendidas, resolvidas, desenvolvidas e avaliadas separadamente. Isso torna os problemas complexos mais fáceis de resolver, as situações novas melhor compreendidas e os grandes sistemas mais fáceis de projetar. Para as séries iniciais, o desenvolvimento desta competência (no escopo da CC) é proposto por meio da identificação e criação de listas de subproblemas para se chegar à solução de um problema maior e o subsequente uso da abstração para lidar com esta tarefa. A decomposição também é uma poderosa técnica de resolução de problemas em matemática. O currículo da matemática propõe a composição e decomposição de números naturais como alternativa para a compreensão de características do sistema de numeração decimal. O processo de composição e decomposição numérica provê uma compreensão da estrutura dos números e do processo envolvido na execução das operações aritméticas básicas. A repetição deste processo, permite a identificação de propriedades algébricas (como fechamento, comutatividade ou associatividade) para as operações, provendo um critério para justificar e avaliar os processos mentais envolvidos nos cálculos. A decomposição de problemas ou números exige uma série de processos cognitivos que exige trabalho e prática para serem desenvolvidos.

Coleta, Organização e Análise de Informações: a coleta, organização e análise de dados são objetos de conhecimento das séries iniciais da Matemática e da Computação. Em particular, na matemática, destacam-se: a leitura, interpretação e representação de dados em listas, tabelas simples ou duplas e gráficos de barras ou colunas; e a coleta, classificação e representação de dados referentes a variáveis categóricas, por meio de tabelas e gráficos. Já nos padrões da CC para o mesmo período, inclui-se também o uso de uma variedade de ferramentas digitais para armazenar, procurar, recuperar, modificar e excluir informações. O gerenciamento de dados no mundo físico e digital é um dos padrões do PC. Foi justamente a necessidade de modelagem e simulação, visualização e gerenciamento de conjuntos maciços de dados que promoveu o surgimento da computação científica. Os computadores geralmente são necessários para encontrar informações em grandes coleções de dados. A computação se preocupa em desenvolver maneiras rápidas e eficientes de se fazer isso. Estas estratégias podem começar a ser trabalhadas desdes as séries iniciais, envolvendo estratégias de organização (como ordenação e busca) e coleta de dados, bem como incluindo diferentes estruturas (por exemplo, gerenciando blocos de montar, conjuntos de provas).

\section{O Pensamento Computacional e a Matemática: benefícios e fundamentos do modelo de integração}

Diversos trabalhos [Freudenthal et al. 2010, Lewis and Shah 2012, da Costa Barbosa et al. 2015, Bean et al. 2015, Love et al. 2016, Lopes et al. 2016] já associaram os conceitos centrais do PC (tais como, algoritmos e procedimentos, simulação, automação, paralelismo, entre outros) com a aprendizagem de conceitos matemáticos. Dentre os benefícios ressaltados no desenvolvimento conjunto entre 
as áreas, destacam-se: o aumento na motivação e interesse no desenvolvimento de conteúdos de ambas as áreas; a ampliação do estudo de conceitos matemáticos pelo uso de fundamentos e recursos computacionais; o incentivo a colaboração, propiciado pelo desenvolvimento de atividades integradoras; o desenvolvimento de habilidades conjuntas; a atração de estudantes a seguir carreira nestas áreas; a melhoria no ensino pela utilização de novas metodologias (oriundas da computação); a apresentação de visões alternativas da matemática tradicional; e a inserção da inovação no ambiente escolar. A concepção do modelo de integração, inspirada nestes benefícios, é fundamentada nos elementos descritos a seguir.

- Construtivismo cognitivo: A proposta de desenvolvimento integrado se fundamenta nos princípios do construtivismo cognitivo de Jean Piaget [Powell and Kalina 2009], que considera que as crianças aprendem construindo sua própria compreensão do mundo quando recebem oportunidades de aprendizagem ativas (por exemplo, experiências e resolução de problemas do mundo real) e onde o professor assume o papel de mediador do conhecimento. Nos primeiros anos de educação, as crianças devem ter experiências significativas, envolvendo descoberta, experimentando e resolvendo problemas desafiadores, com base em seus interesses. A introdução de novos assuntos deve envolver as experiências anteriores dos estudantes, seus interesses ou situações problemáticas diárias, salientando a importância da matemática, da computação e da maneira como elas estão ligadas ao dia-a-dia. Em vez de transmitir informações, o professor deve questionar e desafiar e, em vez de dar as respostas, deve conduzir os alunos a chegarem as próprias conclusões. A tática considerada para a promoção do construtivismo cognitivo é o processo de solução de problemas, o qual deve ser organizado em 4 etapas: (i) definição do problema; (ii) identificação de possíveis soluções; (iii) indicação de soluções alternativas; e (iv) avaliação das alternativas e escolha da solução mais apropriada.

- Aprendizagem ativa: o método de ensino que embasa esta proposta é o da aprendizagem ativa, no qual os estudantes devem participar ativamente do processo de aprendizagem. Embora não exista uma definição precisa para este método, sua aplicação está relacionada com a adoção das seguintes estratégias [Bonwell and Eison 1991]: não tornar os alunos meros ouvintes (eles devem ser os protagonistas no respectivo processo de aprendizagem); dar menos ênfase a transmissão de informação e mais ênfase ao desenvolvimento de habilidades; envolver os estudantes em atividades de discussão, análise, síntese e avaliação; realçar a reflexão dos estudantes sobre suas atitudes. As estratégias consideradas para a inclusão da aprendizagem ativa incluem: (i) o trabalho conjunto, onde os alunos ajudam a responder as perguntas uns dos outros; (ii) a designação de estudantes para apresentar suas soluções para a turma.

- Desenvolvimento gradual: Na proposta do modelo conceitual também é considerada a aprendizagem assistida sugerida por Vygotsky [Vygotsky 1980]. Segundo a sua teoria, os estudantes desenvolvem habilidades de pensamento de alto nível por meio do desenvolvimento gradual. Este desenvolvimento ocorre no processo de solução de problemas quando o professor faz uma orientação dirigida aos alunos (por exemplo, oferecendo dicas e auxílios) ou no trabalho conjunto com colegas capacitados. De acordo com a visão de Vygotsky, estes tipos de interações constituem uma maneira eficaz de desenvolver habilidades e estratégias. Habilidades 
VI Congresso Brasileiro de Informática na Educação (CBIE 2017)

Anais do XXVIII Simpósio Brasileiro de Informática na Educação (SBIE 2017)

estas que os alunos não conseguiriam alcançar sem o auxílio de um adulto ou de colegas. Este nível de desenvolvimento, denominado, zona de desenvolvimento proximal (ZDP) é estabelecido pela diferença entre a capacidade de resolver problemas sem ajuda e a capacidade de resolvê-los com a orientação de uma pessoa mais experiente. O processo para o desenvolvimento gradual inclui: (i) a divisão de uma tarefa em partes menores; (ii) a apresentação de diretrizes e conjunto de passos; (iii) a proposição de feedbacks frequentes.

\section{MaPCom: um modelo conceitual paro o ensino de Matemática integrado ao desenvolvimento do Pensamento Computacional}

O modelo conceitual leva em consideração o desenvolvimento de competências da Matemática para os anos iniciais, a promoção de habilidades do PC e a resolução de problemas. O modelo, ilustrado na Figura 1, inclui a motivação e a introdução de uma unidade matemática por meio de proposição de problemas do dia a dia, o amadurecimento e o o aprofundamento dos conceitos matemáticos envolvidos pela definição de algoritmos e sua síntese por meio da simulação e análise.

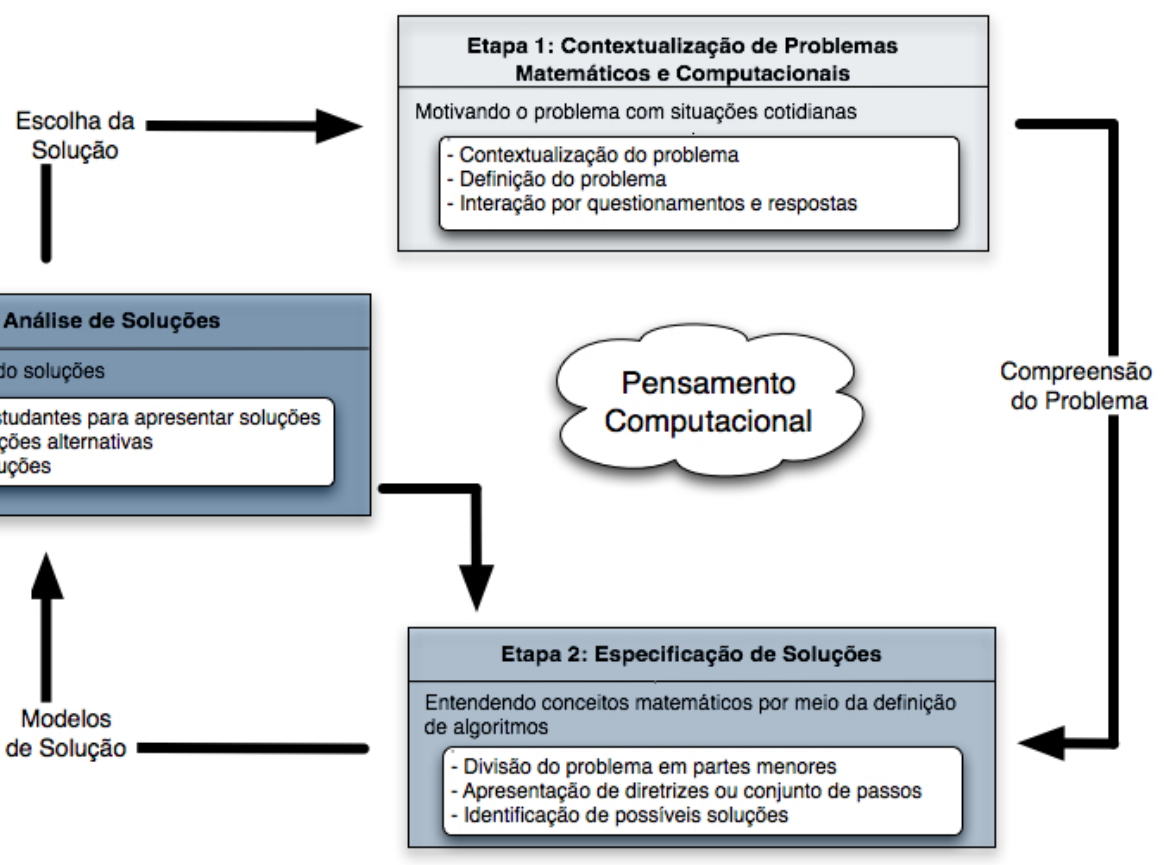

Figura 1. Modelo de Integração

A organização sugerida para o seu planejamento é fundamentada na Taxonomia de Bloom [Bloom 1974], cuja estrutura serve como uma lista de controle para garantir que a formação proposta está incluindo diferentes possibilidades de aprendizagem (nos domínios cognitivo, afetivo ou psicomotor). As habilidades referentes ao domínio cognitivo se relacionam com a compreensão do problema, desde sua contextualização até sua análise crítica. No domínio afetivo as habilidades estão relacionadas às dimensões de ordem afetiva (disposição para responder, participação ativa, compromisso), sendo estimuladas nas discussões introdutórias, na proposição de trabalhos conjuntos e na apresentação de soluções. Já no domínio psicomotor, a manipulação de objetos e os movimentos são es- 
timulados na proposição e simulação das soluções. As etapas envolvidas no planejamento de uma atividade são:

Etapa 1 - Contextualização de Problemas Matemáticos e Computacionais: para introdução e motivação das unidades temáticas (da Matemática e da Computação) a serem trabalhadas, problemas cotidianos que envolvam habilidades comuns de ambas as áreas (por exemplo, envolvendo as competências delineadas na Seção 2) devem ser apresentados. É imprescindível permitir que os alunos indiquem suas próprias considerações e discutam, aprimorando a comunicação, o raciocínio e o desenvolvimento do sentido crítico. Seguindo o guia descrito em [Bonwell and Eison 1991], as habilidades de conhecimento e compreensão podem ser trabalhadas a partir dos seguintes tipos questionamentos:

- Para identificar e relembrar informações:

- Quem, o que, quando, onde, como...? Descreva...

- Para organizar e selecionar fatos ou ideias:

- Relate de novo ou de forma diferente...

Etapa 2 - Especificação de Soluções: o processo de especificar soluções por meio de algoritmos faz com que os estudantes pensem nas etapas que eles (ou um computador) precisam seguir para fazer algo. A definição de um algoritmo relacionado a um conceito matemático exige que o aluno reflita sobre (e compreenda) a natureza do conceito. A discussão e análise das etapas descritas desenvolve a capacidade dos estudantes de argumentar logicamente e a fazer deduções da informação que eles possuem. Observar cuidadosamente as etapas que estabeleceram e usar o raciocínio lógico para explicar o que o algoritmo está realmente fazendo são os pontos de partida para a compreensão ampla e completa de conceitos matemáticos envolvidos. Com as discussões em sala de aula, os professores ganham informações valiosas sobre o pensamento e o progresso dos alunos, enquanto os alunos se tornam mais habilidosos na compreensão da matemática e na comunicação com os outros. Para os níveis de aplicação e análise dos conceitos, os seguintes tipos de questões devem ser considerados [Bonwell and Eison 1991]:

- Para usar os fatos, regras ou princípios:

- Como... é um exemplo de...?

- Como é... relacionado a...?

- Por que... é importante?

- Para separar o todo em partes menores:

- Quais são as partes, características ou componentes de...?

- Classifique... de acordo com...

- Esboce ou faça um diagrama...

- Como... se compara ou contrasta com...?

- Quais evidências você pode listar para...?

A especificação dos (sub-)algoritmos deve ser gradual, podendo envolver as seguintes etapas:

- Descrição dos tipos de entradas e saídas esperados;

- Exemplificação de possíveis entradas e saídas;

- Organização da sequência de passos, a partir de instruções pré-definidas;

- Definição das estruturas de controle a partir de sequências de instruções pré-estabelecidas; 
VI Congresso Brasileiro de Informática na Educação (CBIE 2017)

Anais do XXVIII Simpósio Brasileiro de Informática na Educação (SBIE 2017)

- Especificação completa de soluções algorítmicas.

Etapa 3 - Análise de soluções: a análise crítica sobre o funcionamento de um método, sobre sua generalidade e eficiência ajuda os alunos a entender que a matemática é baseada no senso comum e na razão objetiva. O desenvolvimento desta etapa envolve a designação de estudantes para apresentarem suas soluções, a simulação dos algoritmos pré-definidos, a análise da generalidade das soluções e a indicação de soluções alternativas. Os tipos de questões que podem guiar a etapa de síntese e análise são [Bonwell and Eison 1991]:

- Para combinar as ideais e estabelecer uma visão geral:

- O que você pode inferir de...?

- Quais ideias você pode adicionar para...?

- Como você criaria ou projetaria um novo...?

- O que aconteceria se você combinasse...?

- Quais soluções você sugere para...?

- Para desenvolver opiniões, julgamentos ou decisões:

- Você concorda...? O que você pensa sobre...?

- O que é mais importante...? Estabeleça a ordem de prioridade...

- Como você decidiria sobre...?

- Qual critério você utilizaria para avaliar...?

A concepção de atividades utilizando o MaPCom deve seguir as seguintes orientações:

- Tratar de unidades temáticas da matemática que envolvam o desenvolvimento de habilidades conjuntas do pensamento computacional;

- Estar integradas com o currículo da matemática para o ano correspondente;

- Envolver o trabalho colaborativo em pelo menos uma das etapas;

- Estabelecer momentos de feedbacks em cada uma das etapas;

- Ser planejada com diferentes níveis de dificuldade, de forma que o primeiro nível seja fácil de ser desenvolvido por todos e que o último nível ofereça desafios e permita o desenvolvimento de conceitos avançados.

O planejamento de uma atividade de introdução à multiplicação utilizando o modelo proposto está disponível em http: / / goo.gl/HtMHf3. A estratégia de avaliação indicada para as atividades concebidas por meio do modelo inclui a observação de atitudes em sala de aula, a proposição de desafios e a análise da evolução das soluções apresentadas pelos estudantes. Em particular, para avaliar as habilidades desenvolvidas no domínio cognitivo, sugere-se a elaboração de um conjunto de questões que incluam os seus diversos níveis: conhecimento, compreensão, aplicação, análise, avaliação e criação. Sugere-se que a avaliação contenha pelo menos duas questões de cada nível. A Tabela 1 indica os verbos que podem ser utilizados para elaborar questões para cada um dos níveis.

\section{Considerações Finais}

Neste trabalho é proposto um modelo conceitual para a concepção de atividades que integrem o desenvolvimento de competências da Matemática com a promoção de habilidades do Pensamento Computacional. Os seguintes princípios são observados: a facilidade de domínio e aplicação pelos professores, a integração das atividades com o currículo existente e as vivências cotidianas dos estudantes. 
VI Congresso Brasileiro de Informática na Educação (CBIE 2017)

Anais do XXVIII Simpósio Brasileiro de Informática na Educação (SBIE 2017)

Tabela 1. Níveis da Taxonomia de Bloom e seus respectivos verbos Fonte:[Galhardi and Azevedo 2013]

\begin{tabular}{|l|l|l|l|l|l|}
\hline 1-Lembrar & 2-Entender & 3-Aplicar & 4-Analisar & 5-Avaliar & 6-Criar \\
\hline Reconhecer & Interpretar & Executar & Diferenciar & Verificar & Gerar \\
\hline Relembrar & Exemplificar & Implementar & Organizar & Criticar & Planejar \\
\hline Listar & Classificar & Computar & Atribuir & Julgar & Produzir \\
\hline Nomear & Sumarizar & Resolver & Comparar & Recomendar & Criar \\
\hline Definir & Inferir & Demonstrar & Contrastar & Justificar & Inventar \\
\hline Escrever & Comparar & Utilizar & Separar & Apreciar & Desenvolver \\
\hline Apontar & Explicar & Construir & Categorizar & Ponderar & $\begin{array}{l}\text { Elaborar } \\
\text { hipóteses }\end{array}$ \\
\hline
\end{tabular}

O modelo compreende 3 etapas que podem ser planejadas e executadas de forma circular: contextualização, especificação de soluções algorítmicas e análise e simulação de soluções. Os fundamentos considerados para a concepção do modelo são: a aprendizagem ativa, o construtivismo cognitivo e o desenvolvimento gradual. A ênfase deve ser dada ao desenvolvimento de habilidades (e não na transmissão de informação) e a tática utilizada deve ser o processo de solução (gradual) de problemas.

\section{Referências}

Barbosa, A. and Vale, I. (2014). Early mathematical experiences: the importance of curricular integration. Journal of the European Teacher Education Network, pages $46-56$.

Barcelos, T. S. and Silveira, I. F. (2012). Teaching computational thinking in initial series an analysis of the confluence among mathematics and computer sciences in elementary education and its implications for higher education. In 2012 XXXVIII Conferencia Latinoamericana En Informatica (CLEI), pages 1-8.

Bean, N., Weese, J., Feldhausen, R., and Bell, R. S. (2015). Starting from Scratch: Developing a pre-service teacher training program in computational thinking. In 2015 IEEE Frontiers in Education Conference (FIE), pages 1-8.

Bloom, B. S. (1974). Taxonomy of educational objectives: The classification of educational goals. Handbook 1-2. Longmans: McKay.

Bonwell, C. C. and Eison, J. A. (1991). Active Learning: Creating Excitement in the Classroom. ASHE-ERIC Higher Education Reports.

Bordini, A., Avila, C., Marques, M., Cavalheiro, S., and Foss, L. (2016). Desdobramentos do pensamento computacional no brasil. In Anais do XXVII Simpósio Brasileiro de Informática na Educação (SBIE 2016), pages 200-209.

CSTA and ACM (2016). [INTERIM] CSTA K-12 computer science standards. https: //wWw. csteachers.org/resource/resmgr/Docs/Standards/
2016.standardsRevision/INTERIM_StandardsFINAL_07222.pdf. 
VI Congresso Brasileiro de Informática na Educação (CBIE 2017)

Anais do XXVIII Simpósio Brasileiro de Informática na Educação (SBIE 2017)

da Costa Barbosa, F., Alves, D., Menezes, D., Alexandre, M., Campos, G., Nakamura, Y., Junior, A., and Lopes, C. (2015). Robótica educacional em prol do ensino de matemática. In Anais do XXI Workshop de Informática na Escola (WIE 2015), pages 271-280.

da Silva Rodrigues, R., Andrade, W., Guerrero, D., and Sampaio, L. (2015). Análise dos efeitos do pensamento computacional nas habilidades de estudantes no ensino básico: um estudo sob a perspectiva da programação de computadores. In Anais do XXVI Simpósio Brasileiro de Informática na Educação (SBIE 2015), pages 121-130.

Freudenthal, E., Ogrey, A. N., Roy, M. K., and Siegel, A. (2010). A computational introduction to stem studies. In IEEE EDUCON 2010 Conference, pages 663-672.

Galhardi, A. C. and Azevedo, M. d. (2013). Avaliações de aprendizagem: o uso da taxonomia de bloom. In Workshop de Pós-Graduação e Pesquisa do Centro Paula Souza, volume 8.

Lewis, C. M. and Shah, N. (2012). Building upon and enriching grade four mathematics standards with programming curriculum. In Proceedings of the 43rd ACM technical symposium on Computer Science Education, pages 57-62. ACM.

Lopes, C. C., do Socorro dos Santos Guedes Duarte, M., Sousa, E. A. D., de Souza, R. P., and Pereira, I. B. (2016). O ensino de algoritmos e lógica de programação como uma ferramenta pedagógica para auxiliar a aprendizagem de matemática: Um relato de experiência. In Anais do XXII Workshop de Informática na Escola (WIE 2016), pages 41-50.

Love, B., Winter, V., Corritore, C., and Faimon, D. (2016). Creating an environment in which elementary educators can teach coding. In Proceedings of the The 15th International Conference on Interaction Design and Children, IDC '16, pages 643-648, New York, NY, USA. ACM.

Lu, J. J. and Fletcher, G. H. (2009). Thinking about computational thinking. In Proceedings of the 40th ACM Technical Symposium on Computer Science Education, SIGCSE '09, pages 260-264, New York, NY, USA. ACM.

Ministério da Educação (2016). Base nacional comum curricular. http:// basenacionalcomum.mec.gov.br/images/BNCC_publicacao.pdf.

Powell, K. C. and Kalina, C. J. (2009). Cognitive and social constructivism: Developing tools for an effective classroom. Education, 130(2):241.

Prater, M. A. L. and Mazur, J. M. (2014). Embedded standards-based digital gaming assessments: Pilot study with teachers. In 2014 Computer Games: AI, Animation, Mobile, Multimedia, Educational and Serious Games (CGAMES), pages 1-5.

Vygotsky, L. S. (1980). Mind in society: The development of higher psychological processes. Harvard university press.

Wing, J. M. (2006). Computational thinking. Communications of the ACM, 49(3):33-35. 